\title{
Actinobaculum schaalii - invasive pathogen or innocent bystander? A retrospective observational study
}

\author{
Sarah Tschudin-Sutter ${ }^{1}$, Reno Frei $^{2}$, Maja Weisser ${ }^{1}$, Daniel Goldenberger ${ }^{2}$ and Andreas F Widmer ${ }^{*}$
}

\begin{abstract}
Background: Actinobaculum schaalii is a Gram-positive, facultative anaerobic coccoid rod, classified as a new genus in 1997. It grows slowly and therefore is easily overgrown by other pathogens, which are often found concomitantly. Since 1999, Actinobaculum schaalii is routinely investigated at our hospital, whenever its presence is suspected due to the detection of minute grey colonies on blood agar plates and negative reactions for catalase. The objective of this study was to determine the clinical significance of Actinobaculum schaalii, identified in our microbiology laboratory over the last 11 years.

Methods: All consecutive isolates with Actinobaculum schaalii were obtained from the computerized database of the clinical microbiology laboratory and patients whose cultures from any body site yielded this pathogen were analyzed. Observation of tiny colonies of Gram-positive, catalase-negative coccoid rods triggered molecular identification based on 165 rRNA gene sequencing.

Results: 40 isolates were obtained from 27 patients during the last 11 years. The patient's median age was 81 (19-101) years, 25 (92.6\%) had underlying diseases and 12 (44.4\%) had a genitourinary tract pathology. Actinobaculum schaalii was isolated in 12 urine cultures, 21 blood cultures, and 7 deep tissue biopsies. Twenty-five (62.5\%) specimens were monobacterial, the remaining 15 (37.5\%) were polybacterial 7/7 deep tissue samples (three bloodcultures and five urine cultures). Recovery from urine was interpreted as colonization in 5 (18.6\%) cases (41.6\% of all urine samples). Six (22.2\%) suffered from urinary tract infections, six (22.2\%) from abscesses (skin, intraabdominal, genitourinary tract, and surgical site infections) and 10 (37.0\%) from bacteremia.

Conclusions: In this largest case series so far, detection of Actinobaculum schaalii was associated with an infection - primarily sepsis and abscesses - in $81.5 \%$ of our patients. Since this pathogen is frequently part of polymicrobial cultures (42.5\%) it is often overlooked or considered a contaminant. Detection of Actinobaculum schaalii in clinical isolates mainly reflects infection indicating that this Gram-positive rod is not an innocent bystander.
\end{abstract}

\section{Background}

Actinobaculum schaalii is a Gram-positive, facultative anaerobic, nonmotile coccoid rod, classified as a new genus in 1997 [1]. The genus Actinobaculum includes $A$. schaalii, A. suis, A. massiliae, and A. urinale and is closely related to the genera Actinomyces and Arcanobacterium [1].

Actinobaculum schaalii grows slowly after $48 \mathrm{~h}$ in an anaerobic atmosphere at $37^{\circ} \mathrm{C}$ as tiny grey colonies, less

\footnotetext{
* Correspondence: WidmerA@uhbs.ch

'Division of Infectious Diseases and Hospital Epidemiology, University

Hospital, Basel, Switzerland

Full list of author information is available at the end of the article
}

than $1 \mathrm{~mm}$ in diameter, and shows weak $\beta$-hemolysis on agar plates containing $5 \%$ horse or sheep blood. It is catalase, oxidase, and urease negative and is easily overgrown by other bacteria, which are often found concomitantly. Because of its slow anaerobic growth and resemblance to the normal bacterial flora on skin and mucosa, A. schaalii is often overlooked or considered a contaminant. These difficulties regarding detection and identification impede evaluation of the clinical impact of this pathogen and of its potential to cause invasive infection.

A. schaalii has been reported to be responsible for urinary tract infections, mainly in elderly patients with underlying urological predispositions [2-5]. It has also

\section{Ciomed Central}


been recovered from other human clinical specimens than urine such as blood, but its pathogenic potential remains unknown.

Since 1999, A. schaalii is routinely screened at our hospital. The objective of this study was to determine the clinical significance of $A$. schaalii, identified in our microbiology laboratory over the last 11 years.

\section{Methods}

\section{Setting}

The University Hospital of Basel is an 855 bed tertiary care center in Basel, Switzerland, with approximately $30^{\prime} 000$ admissions per year. The study was approved by the local ethics committee as part of the quality assurance program.

\section{Patients and data collection}

All consecutive isolates with $A$. schaalii were obtained from the computerized database of the clinical microbiology laboratory and patients whose cultures from any body site yielded this pathogen were analyzed. A board certified infectious diseases specialist then reviewed the medical records of these patients and collected data regarding patient demographic characteristics, underlying diseases or condition, clinical manifestations at the time of detection of $A$. schaalii, antibiotic therapy received, and clinical outcome. Definite antibiotic treatment was defined as the antibiotic regimen chosen after identification of the pathogen was completed. Presence of $A$. schaalii was categorized into colonization and infection. Urinary tract infections, surgical site infections, bloodstream infections, bone and joint infections, intraabdominal infections, skin and soft tissue infections, and upper and lower respiratory tract infections were defined according to the criteria of the Centers for Disease Control and Prevention (CDC) [6].

\section{Culture and Species Identification of Actinobaculum schaalii}

Grey, tiny colonies with weak $\beta$-hemolysis or without hemolysis on $5 \%$ Columbia sheep blood agar after $48 \mathrm{~h}$ of anaerobic or $5 \% \mathrm{CO}_{2}$ incubation were further analyzed. All isolates showing Gram-positive coccoid rods and a negative catalase reaction were identified by partial $16 \mathrm{~S}$ RNA gene sequencing using Microseq 500 Bacterial Identification Kit (Applied Biosystems, Rotkreuz, Switzerland) [7-9]. All bacteria growing in specimens supposed to be sterile and monocultures from normally unsterile bodysites were considered to be clinically relevant.

Blood cultures performed at the University Hospital always involve cultivation in an aerobic and in an anaerobic bottle.

\section{Results}

40 specimens with detection of $A$. schaalii were obtained from 27 different patients during the last 11 years. The patient's median age was 81 (19-101) years, and there was a slight male predominance (59.3\%). Twenty-five patients $(92.6 \%)$ had underlying diseases, of which cardiopathy was most commonly encountered (14, 51.9\%). Twelve patients $(44.4 \%)$ had a pathology of the genitourinary tract, mostly prostatic hyperplasia $(5,18.5 \%)$ and five patients (18.5\%) had an urinary catheter or a doubleJ-catheter in place at the time of detection of $A$. schaalii. Only one patient $(3.7 \%)$ was receiving immunosuppressive treatment due to renal transplantation (Table 1).

A. schaalii was detected in 21 blood cultures, 7 deep tissue biopsies and 12 urine cultures. Its presence was interpreted as colonization of the urinary tract without

\section{Table 1 Baseline characteristics and outcome}

\begin{tabular}{lc}
\hline \multicolumn{1}{c}{ Patient's baseline characteristics $(\mathbf{n}=\mathbf{2 7})$ and outcome } \\
\hline Age (years) & \\
Mean & 73 \\
Median & 81 \\
Range & $19-101$ \\
Gender & \\
Male & $16(59.3 \%)$ \\
Female & $11(40.7 \%)$ \\
Hospitalstay (days) & \\
Mean & 18 \\
Median & 9 \\
Range & $1-79$ \\
Immunosuppression & $\mathbf{1}(\mathbf{3 . 7} \%)$ \\
Comorbidities & $\mathbf{2 5}(\mathbf{9 2 . 6 \% )}$ \\
Cancer & $7(25.9 \%)$ \\
Cardiopathy & $14(51.9 \%)$ \\
Chronic renal insufficiency & $4(14.8 \%)$ \\
Diabetes mellitus & $4(14.8 \%)$ \\
Neurological disorder & $13(48.1 \%)$ \\
Hypertension & $6(22.2 \%)$ \\
Chronic pulmonary disorder & $2(7.4 \%)$ \\
Chronic Vascular disorder & $4(14.8 \%)$ \\
Endocriological disorder (other than diabtes mellitus & $3(11.1 \%)$ \\
Chronic pancreatitis & $1(3.7 \%)$ \\
Underlying genitourinary tract pathology & $\mathbf{1 2}(\mathbf{4 4 . 4 \% )}$ \\
Nephrolithiasis & $1(3.7 \%)$ \\
Stenosis & $4(14.8 \%)$ \\
Double-J catheter & $2(7.4 \%)$ \\
Urinary catheter & $3(11.1 \%)$ \\
Prostatic hyperplasia & $5(18.5 \%)$ \\
Renal cysts & $1(3.7 \%)$ \\
Uterine and vaginal prolapse & $1(3.7 \%)$ \\
Renal transplantation & $1(3.7 \%)$ \\
Outcome & \\
Discharge & $17(63.0 \%)$ \\
Referal to another hospital & $3(11.1 \%)$ \\
Referal to a longterm-care facility & $5(18.5 \%)$ \\
Death & $2.4 \%)$ \\
\hline
\end{tabular}


clinical signs or symptoms of infection in five (18.5\%) patients, accounting for $41.6 \%$ of all urine samples. Six patients $(22.2 \%)$ suffered from urinary tract infections, six (22.2\%) from abscesses (skin, intraabdominal, genitourinary tract, and surgical site infections) and 10 (37.0\%) from bacteremia (Table 2).

A. schaalii affected the genitourinary tract infections in most cases (48.1\%). The majority of patients (10/13, $76.9 \%$ ) affected had underlying pathologies of the urinary tract. In three patients with positive blood cultures, diagnosed as urosepsis, Gram-positive rods $\left(10^{4}-10^{6}\right.$ colonies per $\mathrm{ml}$ ) were detected in urine cultures, however, weren't further identified as these cultures were polymicrobial. Abscesses of the genitourinary tract involved the scrotum in one patient and the renal pelvis in another (Table 2).

Surgical site infections occurred in two patients, one after an operation involving the upper jaw, the other after bladder-stone extraction.

Bacteremia with Actinobaculum schaalii was associated with spondylodiscitis and pneumonia, in one case respectively, with intraabdominal infection in three cases and with urinary tract infections in 5 cases (Table 2). In the patient with spondylodiscitis, Actinobaculum schaalii was also detected in the CT-guided biopsy of the disc. Underlying intraabdominal infections detected were occlusive ileus and diverticulitis.

Twenty-three $(57.5 \%)$ specimens were monobacterial, the remaining 17 (42.5\%) were polybacterial 7/7 deep tissue samples, five blood cultures, and five urine cultures (Table 3 ). In patients with polybacterial blood cultures, the other pathogens detected were Pseudomonas aeruginosa ( $P$. aeruginosa in 2 of 2 and $A$. schaalii in 1 of 2 blood cultures) in a patient with urosepsis, Finegoldia magna (Finegoldia magna in 2 of 2 and A. schaalii in 4 of 4 bloodcultures) in a patient with diverticulitis, Clostridium clostridiforme and Bacteroides fragilis (both in 1 of 4 and $A$. schaalii in 1 of 4 blood cultures) in a patient with obstructive ileus and Veillonella (in 2 of 8 and A. schaalii in 8 of 8 bloodcultures) in a patient with unclear intraabdominal infection. Of interest, the patient with $P$. aeruginosa and $A$. schaalii in the blood culture, also had Gram-positive rods $\left(10^{4} \mathrm{CFU} / \mathrm{ml}\right)$ and Gramnegative rods $\left(10^{5} \mathrm{CFU} / \mathrm{ml}\right)$ in the urine culture, however, these weren't further differentiated.

Six patients received no antibiotic treatment, in five of which detection of $A$. schaalii was interpreted as colonization. The remaining patient suffered from an abscess involving the skin, which was surgically drained. Most patients were treated with amoxicillin/clavulanic acid $(10,37.0 \%)$ followed by administration of a quinolone (7, 25.9\%).

Median duration of antibiotic treatment was 15.5 days (range 3 to 84 days). The majority of patients could be discharged $(17,63.0 \%)$, two $(7.4 \%)$ patients died due to metastatic carcinoma, the remaining were transferred to another hospital $(3,11.1 \%)$ or to a long-term-care facility $(5,18.5 \%)$.

\section{Discussion}

A. schaalii caused an invasive infection in $81.5 \%$ of our patients and its presence was interpreted as colonization of the urinary tract in five patients. Infections of the genitourinary tract were by far the most common manifestation of invasive disease. The ability of this pathogen to cause urinary tract infections has also been reported in the literature previously. Beguelin et al. described the clinical features of 20 patients and found urinary tract infections to be the most common manifestation $(11 / 20$, $55 \%$ ) [10]. The authors further described a high rate of underlying urinary tract pathology as detected in our study. We detected A. schaalii in the blood cultures of 10 patients, only half of which were associated with urinary tract infections. The majority of the remaining

Table 2 Distribution of infections and colonisation with Actinobaculum schaalii

\begin{tabular}{|c|c|}
\hline \multicolumn{2}{|c|}{ Infections and colonisation with Actinobaculum schaalii $(n=27)$} \\
\hline Bacteremia & $10(37.0 \%)$ \\
\hline with urinary tract infection & $5(18.5 \%)$ \\
\hline with intaabdominal infection & $3(11.1 \%)$ \\
\hline with spondylodiscitis & $1(3.7 \%)$ \\
\hline with pneumonia & $1(3.7 \%)$ \\
\hline Abscess & $6(22.2 \%)$ \\
\hline Abdominal & $1(3.7 \%)$ \\
\hline Skin & $1(3.7 \%)$ \\
\hline Surgical site infection (upper jaw and after bladder-stone extraction) & $2(7.4 \%)$ \\
\hline Genitourinary tract & $2(7.4 \%)$ \\
\hline Urinary tract infection & $6(22.2 \%)$ \\
\hline with detection of $A$. schaalii in urine culture & $6(22.2 \%)$ \\
\hline Colonisation (detection of $A$. schaalii in urine without clinical signs of infection) & $5(18.6 \%)$ \\
\hline
\end{tabular}


Table 3 Distribution of monobacterial and polybacterial isolates of Actinobaculum schaalii

\begin{tabular}{lc}
\hline \multicolumn{2}{c}{ Specimens with detection of Actinobaculum schaalii $(\mathbf{n}=\mathbf{4 0})$} \\
\hline monobacterial & $23(57.5 \%)$ \\
polybacterial & $17(42.5 \%)$ \\
Urine & $\mathbf{1 2}$ \\
$\quad$ monobacterial & $7(58.3 \%)$ \\
$\quad$ polybacterial & $5(41.7 \%)$ \\
Blood & $\mathbf{2 1}$ \\
$\quad$ monobacterial & $16(76.2 \%)$ \\
polybacterial & $5(23.8 \%)$ \\
Abscess & $\mathbf{7}$ \\
$\quad$ monobacterial & $0(0.0 \%)$ \\
polybacterial & $7(100.0 \%)$ \\
\hline
\end{tabular}

patients had underlying intraabdominal infections and one patient suffered from spondylodiscitis. Therefore, detection of this pathogen in blood cultures should prompt the clinician to search also for abdominal or other foci if no association with the genitourinary tract can be found.

Beguelin et al. described the results of antimicrobial resistance testing in their study, although there are no recognized minimal inhibitory concentration (MIC) interpretative standards for $A$. schaalii [10]. They found that most of their strains showed high MIC levels to ciprofloxacin ( $>32 \mathrm{mg} / \mathrm{L})$ and to trimethoprim/sulfamethoxazole $(>32 \mathrm{mg} / \mathrm{L})$, however, all of their 20 isolated tested, showed low MIC level to beta-lactams ( $\leq$ 0.19 for penicillin) [10]. Similar results were described by Cattoir et al. after testing 48 clinical isolates [11]. The majority of our patients were treated with betalactam-antibiotics, seven however, all with urinary tract infections, were treated with quinolones, possibly without in vitro activity. We therefore conclude, that susceptebilty testing should be routinely performed, when $A$. schaalii is detected in relevant clinical specimens and interpretative standards for interpretation of MICs should be developed. Empirically initiated treatment for urinary tract infections often consists of quinolones or trimethoprim/sulfamethoxazole and may therefore, not be effective.

A. schaalii was often detected together with other bacteria (17 of 40 cultures, $42.5 \%$ ), especially in urine cultures (5 of 12, 41.7\%) and in all abscesses (7 of 7, 100\%). In contrast, Beguelin et al. described a much lower rate of polybacterial cultures, with 18 out of 21 (86\%) specimens being monobacterial [10]. As this pathogen is difficult to detect in the laboratory and is easily overgrown by other bacteria, awareness of its existence is crucial as not to underestimate its presence. Bank et al. analyzed 252 urine samples for presence of $A$. schaalii by a realtime quantitative PCR they developed and found 41 of
252 samples (16\%) to be positive with bacterial concentrations $>10^{4} \mathrm{CFU} / \mathrm{mL}$ [3]. Of 155 urine samples from patients $>60$ years of age, 34 (22\%) were PCR positive, of which 31 (91\%) - the vast majority - harbored other common uropathogenic bacteria, in addition to A. schaalii. In our study three patients with A. schaalii in their blood cultures had Gram-positive rods $\left(10^{4}-10^{6}\right.$ colonies per $\mathrm{ml}$ ) in urine cultures drawn at the same time, however, these weren't further identified as cultures were polymicrobial. One of the patients described in our study with $P$. aeruginosa and A. schaalii in blood cultures, also had Gram-positive rods $\left(10^{4} / \mathrm{ml}\right)$ and Gram-negative rods $\left(10^{5} / \mathrm{ml}\right)$ in the urine culture drawn at the same time. It is plausible that the pathogens detected in the urine culture, were $A$. schaalii and $P$. aeruginosa, respectively, demonstrating that $A$. schaalii has the ability to invade the bloodstream even in the presence of a wellknown uro-pathogen as $P$. aeruginosa.

As our data demonstrate that this pathogen invaded the bloodstream in the presence of other bacteria in primary foci, we conclude that $A$. schaalii is not just an innocent bystander, but has the potential to become an invasive pathogen, leading to severe invasive infection as sepsis, spondylodiscitis [12], and endocarditis [13].

\section{Conclusions}

In this largest case series so far, detection of Actinobaculum schaalii was associated with an infection - primarily sepsis and abscesses - in $81.5 \%$ of our patients. Since this pathogen is frequently part of polymicrobial cultures (42.5\%) it is often overlooked or considered a contaminant. Detection of Actinobaculum schaalii in clinical isolates mainly reflects infection indicating that this Gram-positive rod is not an innocent bystander.

\section{Acknowledgments and Funding}

The University Hospital of Basel funded in part the microbiological analyses of the samples as part of the quality improvement program. No other, especially no commercial funding was received for the study.

\section{Author details}

${ }^{1}$ Division of Infectious Diseases and Hospital Epidemiology, University Hospital, Basel, Switzerland. ${ }^{2}$ Division of Clinical Microbiology, University Hospital, Basel, Switzerland.

\section{Authors' contributions}

All authors read and approved the final manuscript. ST-S wrote the manuscript and analyzed the data RF performed and supervised the microbiological analyses. MW supervised and edited the manuscript. DG performed and supervised the microbiological analyses. AFW initiated the study, analysed the data, supervised the whole project and edited the manuscript.

\section{Competing interests}

The authors declare that they have no competing interests.

Received: 28 March 2011 Accepted: 26 October 2011

Published: 26 October 2011 


\section{References}

1. Lawson PA, Falsen E, Akervall E, Vandamme P, Collins MD: Characterization of some Actinomyces-like isolates from human clinical specimens: reclassification of Actinomyces suis (Soltys and Spratling) as Actinobaculum suis comb. nov. and description of Actinobaculum schaalii sp. nov. Int J Syst Bacteriol 1997, 47:899-903.

2. Reinhard M, Prag J, Kemp M, Andresen K, Klemmensen B, Højlyng N, Sørensen SH, Christensen JJ: Ten cases of Actinobaculum schaalii infection: clinical relevance, bacterial identification, and antibiotic susceptibility. J Clin Microbiol 2005, 43:5305-8.

3. Bank S, Jensen A, Hansen TM, Søby KM, Prag J: Actinobaculum schaalii, a common uropathogen in elderly patients, Denmark. Emerg Infect Dis 2010, 16:76-80.

4. Nielsen HL, Søby KM, Christensen JJ, Prag J: Actinobaculum schaalii: a common cause of urinary tract infection in the elderly population. Bacteriological and clinical characteristics. Scand J Infect Dis 2010, 42:43-7.

5. Fendukly F, Osterman B: Isolation of Actinobaculum schaalii and Actinobaculum urinale from a patient with chronic renal failure. J Clin Microbiol 2005, 43:3567-9

6. Garner JS, Jarvis WR, Emori TG, Horan TC, Hughes JM: CDC definitions for nosocomial infections, 1988. Am J Infect Control 1988, 16:128-40.

7. Wilson KH, Blichington RB, Greene RC: Amplification of bacterial $16 \mathrm{~S}$ ribosomal DNA with polymerase chain reaction. J Clin Microbiol 1990, 28:1942-6.

8. Drancourt M, Berger P, Raoult D: Systematic 16S rRNA. Gene sequencing of atypical clinical isolates identified 27 new bacterial species associated with humans. J Clin Microbiol 2004, 42:2197-202.

9. Bosshard P, Abels S, Zbinden R, Böttger EC, Altwegg M: Ribosomal DNA sequencing for identification of aerobic gram-positive rods in the clinical laboratory (an 18-month evaluation). J Clin Microbiol 2003, 41:4134-40.

10. Beguelin C, Genne D, Vara A, Tritten ML, Siegrist HH, Jaton K, Lienhard R: Actinobaculum schaalii: clinical observation of 20 cases. Clin Microbiol Infect 2010.

11. Cattoir V, Varca A, Greub G, Prod'hom G, Legrand P, Lienhard R: In vitro susceptibility of Actinobaculum schaalii to 12 antimicrobial agents and molecular analysis of fluoroquinolone resistance. J Antimicrob Chemother 2010, 65:2514-7.

12. Haller P, Bruderer $T$, Schaeren $S$, Laifer $G$, Frei R, Battegay M, Flückiger U, Bassetti S: Vertebral osteomyelitis caused by Actinobaculum schaalii: a difficult-to-diagnose and potentially invasive uropathogen. Eur I Clin Microbiol Infect Dis 2007, 26:667-70.

13. Hoenigl M, Leitner E, Valentin T, Zarfel G, Salzer HJ, Krause R, Grisold AJ: Endocarditis caused by Actinobaculum schaalii, Austria. Emerg Infect Dis 2010, 16:1171-3.

\section{Pre-publication history}

The pre-publication history for this paper can be accessed here: http://www.biomedcentral.com/1471-2334/11/289/prepub

doi:10.1186/1471-2334-11-289

Cite this article as: Tschudin-Sutter et al:: Actinobaculum schaalii -

invasive pathogen or innocent bystander? A retrospective observational study. BMC Infectious Diseases 2011 11:289.

\section{Submit your next manuscript to BioMed Central and take full advantage of:}

- Convenient online submission

- Thorough peer review

- No space constraints or color figure charges

- Immediate publication on acceptance

- Inclusion in PubMed, CAS, Scopus and Google Scholar

- Research which is freely available for redistribution

Submit your manuscript at www.biomedcentral.com/submit 